\title{
SYMBOLIC POWERS OF PRIME IDEALS AND THEIR TOPOLOGY
}

\author{
PETER SCHENZEL
}

\begin{abstract}
For a prime ideal $P$ of a commutative Noetherian ring $R$ a necessary and sufficient condition is given to determine when the $P$-adic topology is equivalent, resp. linearly equivalent, to the $P$-symbolic topology. The last means that the symbolic Rees ring is a finitely generated module over the ordinary Rees ring of $P$. Then it is considered when the integral closure of all the powers of $P$ are primary.
\end{abstract}

1. Introduction and main results. Let $R$ denote a commutative Noetherian ring. For a prime ideal $P$ of $R$ we define $P^{(n)}=P^{n} R_{P} \cap R$, the $n$th symbolic power of $P$. Note that $P^{(n)}$ is equal to the uniquely determined $P$-primary component of $P^{n}$. In [3, §7], R. Hartshorne writes: "A general question, whose solution is quite complicated, is to determine when the $P$-adic topology is equivalent to the $P$-symbolic topology." Here the $P$-symbolic topology denotes the topology defined by the symbolic powers $P^{(n)}, n \geqslant 1$, of $P$. In the following we shall give a complete solution to this problem. For an arbitrary ideal $I$ of $R$ note that the sets Ass $R / I^{n}$ stabilize for large $n$ (see M. Brodmann [1]). So we denote this stabilized set by $A^{*}(I)$.

THEOREM 1. For a prime ideal $P$ of a commutative Noetherian ring $R$ the following conditions are equivalent:

(i) The $P$-adic topology is equivalent to the $P$-symbolic topology.

(ii) height $\left(\widehat{P R_{Q}}+p / p\right)<\operatorname{dim} \widehat{R_{Q}} / p$ for all $Q \in A^{*}(P) \backslash\{P\}$ and $p \in$ Ass $\widehat{R_{Q}}$.

Here $\widehat{R_{Q}}$ denotes the $Q R_{Q}$-adic completion of $R_{Q}$. A particular case of Theorem 1 has been shown by R. Hartshorne [3, Proposition 7.1]. Let $P$ be a prime ideal satisfying condition (i) of the previous theorem. Then, for every integer $n \geqslant 1$ there is an integer $m \geqslant n$ such that $P^{(m)} \subseteq P^{n}$. Now the question is whether there exists an integer $k$ such that $P^{(n+k)} \subseteq P^{n}$ for all $n \geqslant 1$. In this case we say that the $P$-adic topology is linearly equivalent to the $P$-symbolic topology. It is equivalent to the fact that the "symbolic" Rees ring $\oplus_{n \geqslant 0} P^{(n)}$ is a finitely generated graded module over $\oplus_{n \geqslant 0} P^{n}$, the ordinary Rees ring of $P$. For our next result let us recall the notion of analytic spread $a(I)$ of an ideal $I$ of a local Noetherian ring $(R, M)$, i.e., $a(I)=$ $\operatorname{dim} R(I) \otimes_{R} R / M$, where $R(I)=\oplus_{n \geqslant 0} I^{n}$ denotes the Rees ring of $R$ with respect to $I$. Note that height $I \leqslant a(I) \leqslant \operatorname{dim} R$.

Received by the editors October 13, 1983 and, in revised form, February 6, 1984.

1980 Mathematics Subject Classification. Primary 13A17; Secondary 13C15, 13H10.

Key words and phrases. Prime ideal, symbolic power, asymptotic prime divisors, integral closure, Rees ring, form ring. 
ThEOREM 2. For a prime ideal $P$ of a commutative Noetherian ring $R$ the following conditions are equivalent:

(i) The P-adic topology is linearly equivalent to the P-symbolic topology.

(ii) $a\left(P \widehat{R_{Q}}+p / p\right)<\operatorname{dim} \widehat{R_{Q}} / p$ for all $Q \in A^{*}(P) \backslash\{P\}$ and $p \in \operatorname{Ass} \widehat{R_{Q}}$.

If one of these conditions is satisfied it follows:

(iii) $A_{a}^{*}(P)=\{P\}$.

Moreover, if $R$ is locally unmixed all of the above conditions are equivalent.

Here let $I_{a}$ denote the integral closure of an ideal $I$ of $R$, i.e., the set of elements $x \in R$ satisfying an equation $x^{m}+c_{1} x^{m-1}+\cdots+c_{m}=0, c_{i} \in I^{i}, i=1, \ldots, m$. Then Ass $R /\left(I^{n}\right)_{a}$ stabilizes for large $n$, see L. J. Ratliff, Jr. [8]. This stabilized set is denoted by $A_{a}^{*}(I)$. A local ring $(R, M)$ is called unmixed if $\operatorname{dim} \hat{R} / p=\operatorname{dim} R$ for all $p \in$ Ass $\hat{R}$, where $\hat{R}$ denotes the $M$-adic completion. $R$ is called locally unmixed if $R_{Q}$ is unmixed for all prime ideals $Q$ of $R$.

Corollary 1. Let $P$ denote a prime ideal of a locally unmixed Noetherian ring $R$. Then the following conditions are equivalent:

(i) There is an integer $k$ such that $P^{(n+k)} \subseteq P^{n}$ for all $n \geqslant 1$,

(ii) $a\left(P R_{Q}\right)<\operatorname{dim} R_{Q}$ for all $Q \in A^{*}(P) \backslash\{P\}$, and

(iii) Ass $R /\left(P^{n}\right)_{a}=\{P\}$ for all $n \geqslant 1$.

The question whether Ass $R /\left(P^{n}\right)_{a}=\{P\}$ for all $n \geqslant 1$ is a variation of the problem when does Ass $R / P^{n}=\{P\}$ for all $n \geqslant 1$, which has received some previous attention. It is a well-known fact that $\operatorname{gr}_{R}(P)$ is a domain if and only if $\operatorname{gr}_{R_{P}}\left(P R_{P}^{\prime}\right)$ is a domain and Ass $R / P^{n}=\{P\}$ for all $n \geqslant 1$. Here $\operatorname{gr}_{R}(I)=$ $\oplus_{n \geqslant 0} I^{n} / I^{n+1}$ denotes the form ring of $R$ with respect to an ideal $I$ of $R$.

THeOREM 3. Let $P$ denote a prime ideal of a commutative Noetherian ring $R$. Then $\operatorname{gr}_{R}(P)_{\text {red }}$ is a domain if and only if $\operatorname{gr}_{R_{P}}\left(P R_{P}\right)_{\text {red }}$ is a domain and $A_{a}^{*}(P)=\{P\}$.

If $R$ is a commutative Noetherian ring, by $R_{\text {red }}$ we denote the reduced ring of $R$, i.e., $R$ modulo its nilradical. Hence, $A_{a}^{*}(P)=\{P\}$ is, in a certain sense, a transitivity condition. Note that particular cases of Theorem 3 were obtained by $C$. Huneke [4]. Another result related to the question when does $A_{a}^{*}(P)=\{P\}$ has been shown by S. McAdam in [7].

2. Proof of Theorem 1. Before we shall give the proof, let us fix notation. For two ideals $I, J$ in a Noetherian ring $R$ we define $I:\langle J\rangle=\bigcup_{k \geqslant 1} I: J^{k}$. Of course $I:\langle J\rangle=I: J^{k}$ for sufficiently large $k$.

(i) $\Rightarrow$ (ii): Assume the contrary. Then there are a prime ideal $Q \in A^{*}(P) \backslash\{P\}$ and a prime ideal $p \in$ Ass $\widehat{R_{Q}}$ such that $\operatorname{dim} \widehat{R_{Q}} /\left(R \widehat{R_{Q}}+p\right)=0$. By changing the notation we may assume $(R, M)$ a local ring and $\operatorname{dim} \hat{R} /(P \hat{R}+p)=0$ for some $p \in$ Ass $\hat{R}$. Then $0 \neq 0:\langle p\rangle$, because $p \in$ Ass $\hat{R}$. On the other hand

$$
P^{n} \hat{R}:\langle p\rangle=P^{n} \hat{R}:\langle P \hat{R}+p\rangle=P^{n} \hat{R}:\langle M\rangle \subseteq P^{(n)} \hat{R}
$$

because $(P \hat{R}+p)^{2 k} \subseteq P^{k} \hat{R}+p^{k} \subseteq(P \hat{R}+p)^{k}$ for some integer $k \geqslant 1$ and because $P \hat{R}+p$ is $M$-primary. Therefore it follows that $0 \neq 0:\langle p\rangle \subseteq \bigcap_{n \geqslant 1} P^{(n)} \hat{R}$, which is a contradiction to (i). 
(ii) $\Rightarrow$ (i): Set $E_{m n}=P^{(m)}+P^{n} / P^{n}$ for $m, n \geqslant 1$. Because

$$
\text { Ass } E_{m n} \subseteq A^{*}(P) \backslash\{P\}
$$

it is enough to show the statement in any $R_{Q}, Q \in A^{*}(P) \backslash\{P\}$. That is, we may assume $R$ a local ring with $M$ its maximal ideal. Now we make an induction on $\operatorname{dim} R / P$. First assume $\operatorname{dim} R / P=1$. For a fixed integer $n, E_{m n}$ becomes a decreasing sequence of modules of finite length. Hence, it becomes stationary. Set $E_{n}=P^{(m(n))}+P^{n}$, where $m(n)$ is an integer such that $E_{m n}=E_{m(n), n}$ for all $m \geqslant$ $m(n)$. It follows that $E_{n}=E_{n+1}+P^{n}$ for all $n$. Now assume $E_{k} \neq P^{k}$ for some $k$. So we may choose elements $x_{n} \in E_{n} \backslash P^{n}$ for all $n \geqslant k$ such that $x_{n+1} \equiv x_{n} \bmod P^{n}$. Then $\left\{x_{n}\right\}$ defines a nonzero element of $\lim _{\leftarrow} E_{n} / P^{n}$. Therefore it follows that

$$
0 \neq \bigcap_{n \geqslant 0} E_{n}=\lim _{n} E_{n} / P^{n} \subseteq \lim _{n} \hat{R} / P^{n} \hat{R} \cong \hat{R},
$$

because $E_{n} / P^{n} \cong E_{n} / P^{n} \otimes_{R} \hat{R}$ is an $R$-module of finite length. Next we choose an element $x \in \bigcap_{n \geqslant 0} E_{n}$ such that $0: x=p$ for some associated prime ideal $p$ of $\hat{R}$. By the Artin-Rees Lemma there exists an integer $r \geqslant k$ such that $P^{r} \hat{R} \cap x \hat{R} \subseteq x P \hat{R}$. Since $x \in E_{r}$ and $E_{r} / P^{r}$ has finite length, $M^{s} x \subseteq P^{r} \hat{R} \cap x \hat{R} \subseteq x P \hat{R}$ for some $s \geqslant 1$. Then $M^{s}+p \subseteq P \hat{R}+p$, which contradicts (ii). Second suppose $\operatorname{dim} R / P>1$. As above we consider $E_{m n}$. As the localizations of these modules in any prime ideal $Q \in A^{*}(P) \backslash\{P\}$ with $Q \neq M$ tend to zero by induction, they have finite length for $m$ large. Hence, Ass $E_{m n} \subseteq V(M)$ for $m$ large. Now the proof follows as in the case $\operatorname{dim} R / P=1$. Hence we omit it.

REMARK. It follows from the proof of Theorem 1 that we may replace (ii) in Theorem 1 by the following condition:

(ii)' height $\left(P \widehat{R_{Q}}+p / p\right)<\operatorname{dim} \widehat{R_{Q}} / p$ for all $Q \in V(P) \backslash\{P\}$ and $p \in$ Ass $\widehat{R_{Q}}$.

Here $V(P)$ denotes the set of prime ideals containing $P$.

3. Proof of Theorem 2. To this end we have to recall some notation. For an arbitrary ideal $I$ of $R$ we denote by $R(I)=\oplus_{n \geqslant 0} I^{n}$ the Rees ring of $R$ with respect to $I$. A filtration $\left\{I_{n}\right\}_{n \geqslant 0}$ of ideals of $R$ is called $I$-good if $I I_{n} \subseteq I_{n+1}$ for all $n \geqslant 0$. For an $I$-good filtration $\left\{I_{n}\right\}_{n \geqslant 0}$ of $R$ we may form the graded $R(I)$-module $\oplus_{n \geqslant 0} I_{n}$. Note that it is finitely generated if and only if there is an integer $k$ such that $I_{n+k} \subseteq I^{n}$ for all $n \geqslant 1$. Now let $(R, M)$ be a local ring. As the relations

$$
\left(I^{n}:\langle M\rangle\right)\left(I^{m}:\langle M\rangle\right) \subseteq I^{n+m}:\langle M\rangle
$$

hold, we may form the graded $R(I)$-algebra $S(I)=\oplus_{n \geqslant 0} I^{n}:\langle M\rangle$. We are interested in a finiteness criterion of $S(I)$ over $R(I)$. To this end we are looking at the $M$-transform $D_{M}(\cdot)=\lim _{n} \operatorname{Hom}_{R}\left(M^{n}, \cdot\right)$ defined on $R$-modules. If depth $R>0$ we have

$$
D_{M}\left(I^{n}\right) D_{M}\left(I^{m}\right) \subseteq D_{M}\left(I^{n+m}\right) \quad \text { and } \quad I^{n}:\langle M\rangle \subseteq D_{M}\left(I^{n}\right),
$$

as easily seen. $D_{M}\left(I^{n}\right)$ is an ideal of $D_{M}(R)$. Thus we may define the graded $S(I)$-algebra $D(I)=\bigoplus_{n \geqslant 0} D_{M}\left(I^{n}\right)$, occuring together with the canonical injection $S(I) \rightarrow D(I)$. It follows easily that $D(I) \cong D_{M R(I)}(R(I))$. The next result is the crucial point in order to prove Theorem 2 . 
Proposition. Let $(R, M)$ denote a local Noetherian ring. For an ideal $I$ of $R$ the following conditions are equivalent:

(i) $D(I)$ is finitely generated over $R(I)$ and depth $R>0$,

(ii) $S(I)$ is finitely generated over $R(I)$, and

(iii) $a(I \hat{R}+p / p)<\operatorname{dim} \hat{R} / p$ for all $p \in$ Ass $\hat{R}$.

Proof. The implication (i) $\Rightarrow$ (ii) holds trivially because of the canonical injection $S(I) \rightarrow D(I)$. Now let us show (ii) $\Rightarrow$ (iii). According to (ii) there is an integer $k$ such that $I^{n+k}:\langle M\rangle \subseteq I^{n}$ for all $n \geqslant 1$. Let $p \in$ Ass $\hat{R}$ and $p=0: x$ for some $x \in \hat{R}$. By the Artin-Rees lemma there is an integer $s$ such that $I^{n+s} \hat{R} \cap x \hat{R} \subseteq x I^{n} \hat{R}$ for all $n \geqslant 1$. Then $I^{n+k+s}(\hat{R} / p):\langle M\rangle \subseteq I^{n}(\hat{R} / p)$ for all $n \geqslant 1$ as easily seen. That is, without loss of generality we may assume $R$ a complete integral domain. According to D. Rees [10, Theorem 1.4], there is an integer $t$ such that $\left(I^{n+t}\right)_{a} \subseteq I^{n}$ for all $n \geqslant 1$. Thus there is an integer $r$ such that $\left(I^{n+r}\right)_{a}:\langle M\rangle \subseteq I^{n}$ for all $n \geqslant 1$. Therefore, $S_{a}(I)=\bigoplus_{n \geqslant 0}\left(I^{n}\right)_{a}:\langle M\rangle$ is a finitely generated graded $R(I)$-module. Let $R_{a}(I)=\bigoplus_{n \geqslant 0}\left(I^{n}\right)_{a}$. Then it follows that

$$
R(I) \subseteq R_{a}(I) \subseteq S_{a}(I) \subseteq R[T],
$$

where $T$ denotes an indeterminate over $R$. Because $R_{a}(I)$ is the integral closure of $R(I)$ in $R[T]$ the finiteness of $S_{a}(I)$ over $R(I)$ yields $R_{a}(I)=S_{a}(I)$, i.e., $\left(I^{n}\right)_{a}=$ $\left(I^{n}\right)_{a}:\langle M\rangle$ for all $n \geqslant 1$. Hence, $M \notin A_{a}^{*}(I)$. Because $R$ satisfies the altitude formula it follows that $a(I)<\operatorname{dim} R$ by virtue of S. McAdam [6, Theorem 3]. In order to prove (iii) $\Rightarrow$ (i) we first note that it is enough to show that $D(I \hat{R})$ is finitely generated over $R(I \hat{R})$. Second it is enough to show that $D(I(\hat{R} / p))$ is finitely generated over $R(I(\hat{R} / p)$ ) for all $p \in$ Ass $\hat{R}$ (see M. Brodmann [2, (3.3) and (6.2)]). Without loss of generality we may assume height $(I \hat{R}+p / p)>0$. Because

$$
a(I \hat{R}+p / p)=\operatorname{dim} R(I(\hat{R} / p)) \otimes_{\hat{R}} \hat{R} / M,
$$

condition (iii) yields height $M R(I(\hat{R} / p))>1$ according to the altitude formula. Then the statement follows by virtue of M. Brodmann $[2,(6.3)]$.

Corollary 2. Let $(R, M)$ be an unmixed local ring. Then the following conditions are equivalent:

(i) There is an integer $k$ such that $I^{n+k}:\langle M\rangle \subseteq I^{n}$ for all $n \geqslant 1$.

(ii) $a(I)<\operatorname{dim} R$.

Proof. According to the Proposition it is enough to show that $a(I)<\operatorname{dim} R$ is equivalent to condition (iii). Because $R$ is unmixed we have $\operatorname{dim} R=\operatorname{dim} \hat{R} / p$ for all $p \in$ Ass $\hat{R}$. According to L. J. Ratliff, Jr. [9, (9.2)], we get $a(I \hat{R}+p / p) \leqslant a(I)$ for all minimal prime ideals $p \in$ Ass $\hat{R}$ and equality holds for some of them.

Corollary 2 answers a question of the author in a preliminary version of [11]. Independently it was shown by D. Katz in [5] and in a private communication.

Proof of TheOREM 2. (i) $\Rightarrow$ (ii): If there is an integer $k \geqslant 1$ such that $P^{(n+k)} \subseteq$ $P^{n}$ for all $n \geqslant 1$, it yields $P^{n+k} R_{Q}:\left\langle Q R_{Q}\right\rangle \subseteq P^{(n+k)} R_{Q} \subseteq P^{n} R_{Q}$ for all $Q \in$ $A^{*}(P) \backslash\{P\}$. That is, $S\left(P R_{Q}\right)$ is finitely generated over $R\left(P R_{Q}\right)$. Hence the conclusion follows by virtue of the Proposition. 
(ii) $\Rightarrow$ (i): Without loss of generality we may assume $(R, M)$ a local ring as follows by passing to $R_{Q}, Q \in A^{*}(P) \backslash\{P\}$. Now we make an induction on $\operatorname{dim} R / P$. First let $\operatorname{dim} R / P=1$. So it is enough to show that there is an integer $k$ such that $P^{n+k}:\langle M\rangle \subseteq P^{n}$ for all $n \geqslant 1$. Hence, the statement follows from the Proposition. Second let $\operatorname{dim} R / P>1$. By induction we may assume that there is an integer $k$ such that $P^{(n+k)} \subseteq P^{n}:\langle M\rangle$ for all $n \geqslant 1$. Note that the claim is true in any localization $Q \in A^{*}(P) \backslash\{P, M\}$. If $M \notin A^{*}(P)$, there is nothing to show. Otherwise, it is enough to prove that there is an integer $r$ with $P^{n+r}:\langle M\rangle \subseteq P^{n}$ for all $n \geqslant 1$. This is true by virtue of the Proposition.

(ii) $\Rightarrow$ (iii): Since $\widehat{R_{Q}} / p$ satisfies the altitude formula it follows that $Q\left(\widehat{R_{Q}} / p\right) \notin$ $A_{a}^{*}\left(P\left(\widehat{R_{Q}} / p\right)\right)$ for all $Q \in A^{*}(P) \backslash\{P\}$ and $p \in$ Ass $\widehat{R_{Q}}$ (see S. McAdam [6, Theorem 3]). By virtue of L. J. Ratliff, Jr. [9, (8.3)], we get $\widehat{Q R_{Q}} \notin A_{a}^{*}\left(P \widehat{R_{Q}}\right)$ and $Q \notin A_{a}^{*}(P)$ for all $Q \in A^{*}(P) \backslash\{P\}$. Since $A_{a}^{*}(P) \subseteq A^{*}(P)$ (see L. J. Ratliff, Jr. [8, (2.6.1)]), we have $A_{a}^{*}(P)=\{P\}$ as required. If $R$ is locally unmixed all of the above conclusions are reversible, i.e., (iii) $\Rightarrow$ (ii).

Proof of Corollary 1. It uses the same circle of ideas as given in the proof of Corollary 2, where we have to note that Ass $R /\left(I^{n}\right)_{a} \subseteq$ Ass $R /\left(I^{n+1}\right)_{a}$ (see [8]).

4. Proof of Theorem 3. Before we shall embark in the proof we have to recall a well-known fact. Let $Q$ denote a prime ideal containing $P$ and $S=R \backslash Q$. Then $\operatorname{gr}_{R_{Q}}\left(P R_{Q}\right) \cong \operatorname{gr}_{R}(P)_{S^{*}}$, where $S^{*}$ denotes the set of leading forms of elements of $S$ in $\operatorname{gr}_{R}(P)$. First assume $\operatorname{gr}_{R}(P)_{\text {red }}$ a domain. Since it is stable under localization, it is enough to show $A_{a}^{*}(P)=\{P\}$. By localization we see that it is enough to assume $R$ is local with maximal ideal $M$ and to show that it forces $M \notin A_{a}^{*}(P)$. Assume the contrary. Then we may construct an element $x \in R$ such that $x^{*}$, the initial form of $x$ in $\operatorname{gr}_{R}(P)$, is not nilpotent, $x \notin\left(P^{n}\right)_{a}$ and $M x \subseteq\left(P^{n}\right)_{a}$ for some $n$. Choose $k$ such that $x \in P^{k} \backslash P^{k+1}$. Note that $n \geqslant k+1$. For an element $y \in M \backslash P$ it yields $x y \in\left(P^{n}\right)_{a}$, i.e., $(x y)^{m}+c_{1}(x y)^{m-1}+\cdots+c_{m}=0$ for a certain integer $m$ and $c_{i} \in P^{n i}$. Thus, $(x y)^{m} \in P^{m k+1}$ and $x^{*} y^{*}$ is nilpotent. Because $y^{*}$ is not nilpotent this is a contradiction. In order to prove the converse we first note that $A_{a}^{*}(P)=\{P\}$ yields $P^{(n)} \subseteq\left(P^{n}\right)_{a}$ for all $n \geqslant 1$, as easily seen. Now suppose there are elements $x, y$ such that $x^{*} y^{*}$ is nilpotent, while $y^{*}$ is not nilpotent. $\operatorname{Because} \operatorname{gr}_{R_{p}}\left(P R_{p}\right) \cong$ $\operatorname{gr}_{R}(P)_{S^{*}}, S=R \backslash P$, and $\operatorname{gr}_{R_{P}}\left(P R_{P}\right)_{\text {red }}$ is a domain, the assumption yields that there is an $s \in R \backslash P$ such that $s^{*} x^{*}$ is nilpotent. Let $x \in P^{t} \backslash P^{t+1}$. Then it follows that $s^{k} x^{k} \in P^{t k+1}$ for a certain $k \geqslant 0$. This yields $x^{k} \in P^{(t k+1)} \subseteq\left(P^{t k+1}\right)_{a}$ and $x^{k m} \in P^{t k m+1}$ for a certain integer $m$. Therefore $x^{*}$ is nilpotent, as required.

Corollary 3. Let $P$ be a prime ideal of a commutative Noetherian ring $R$. Suppose $\operatorname{gr}_{R_{P}}\left(P R_{P}\right)$ is a domain (e.g., $R_{P}$ is a regular local ring). Then $\operatorname{gr}_{R}(P)_{\text {red }}$ is a domain if and only if $P^{(n)}=\left(P^{n}\right)_{a}$ for all $n \geqslant 1$.

Proof. By virtue of Theorem 3 it is enough to show the "only if" part. Because $A_{a}^{*}(P)=\{P\}$ and $P^{(n)} \subseteq\left(P^{n}\right)_{a}$, see Theorem 3, we may consider the natural injection $\left(P^{n}\right)_{a} / P^{(n)} \rightarrow R / P^{(n)}$. Therefore it is enough to show $\left(P^{n}\right)_{a} R_{p}=P^{n} R_{P}$. But this follows easily because $\operatorname{gr}_{R_{P}}\left(P R_{P}\right)$ is a domain. 


\section{REFERENCES}

1. M. Brodmann, Asymptotic stability of Ass $\left(M / I^{n} M\right)$, Proc. Amer. Math. Soc. 74 (1979), 16-18.

2. , Finiteness of ideal transform, J. Algebra 63 (1980), 162-185.

3. R. Hartshorne, Affine duality and cofiniteness, Invent. Math. 9 (1970), 721-737.

4. C. Huneke, On the associated graded ring of an ideal, Illinois J. Math. 26 (1982), 121-137.

5. D. Katz, Prime divisors, asymptotic $R$-sequences and unmixed local rings, preprint.

6. S. McAdam, Asymptotic prime divisors and analytic spreads, Proc. Amer. Math. Soc. 80 (1980), 555-558.

7. __ Asymptotic prime divisors and going down, Pacific J. Math. 91 (1980), 179-186.

8. L. J. Ratliff, Jr., On prime divisors of $I^{n}$, $n$ large, Michigan Math. J. 23 (1976), 337-352.

9. __ On asymptotic prime divisors, Pacific J. Math. 111 (1984), 395-413.

10. D. Rees, A note on analytically unramified local rings, J. London Math Soc. 36 (1961), 24-28.

11. P. Schenzel, Independent elements, unmixedness theorems and asymptotic prime divisors, J. Algebra (to appear).

Sektion Mathematik der Martin - Luther - Universität, DDR - 4010 Halle, German Dem OCRATIC REPUBLIC 